\title{
Sentiments d'injustice, conceptions du juste et positionnement politique
}

\author{
Feelings of injustice, conceptions of justice and \\ political preferences
}

CAROLINE GUIBET LAFAYE***

\begin{abstract}
Résumé: L'enquête menée en France en 20102011 sur le thème Perception des inégalités et sentiments de justice a mis en évidence le rôle structurant de l'opposition gauche/droite sur les conceptions de la justice. Une hypothèse explicative de cette incidence réside dans la cohérence intrinsèque des représentations morales du juste. Néanmoins l'hétérogénéité des sentiments d'injustice et la variabilité des attitudes à l'égard des inégalités suggèrent plusieurs pistes interprétatives: y a-t-il une identité sociale ou politique chez les individus qui tendent à accepter ou rejeter les inégalités? Les positions sociales et les préférences politiques ont-elle une incidence sur la tolérance aux inégalités?
\end{abstract}

Mots clés: Sentiment d'injustice, inégalités, préférences politiques, mérite, volontarisme.

\begin{abstract}
A survey by questionnaire and interviews conducted in 2010-2011 in France on «Perception of Inequalities and Feelings of Justice» (PISJ) highlighted the structuring - even prominent - role of the French left/ right (liberal vs. conservative) opposition on the conceptions of justice and on the endorsement of principles of distributive justice. An explanatory hypothesis of this influence is to be found in the intrinsic coherence of moral representations (of the just). Is there a social, political identity and/or other common traits among individuals who tend to accept or, conversely, to reject inequalities? Do social positions and political preferences have an impact on the tolerance of inequality?
\end{abstract}

Key words: Feelings of injustice, inequalities, political preferences, merit, voluntarism.

Une enquête, par questionnaire et entretiens semi-directifs menée en France en 20102011 sur le thème Perception des inégalités et sentiments de justice (PISJ), a mis en évidence le rôle structurant - voire prééminent eu égard à d'autres caractéristiques sociales - de l'opposition gauche/droite sur les conceptions de la justice et de l'adoption de principes de

Fecha de recepción: 09/ 12/ 2012. Fecha de aceptación: 23/ 02/ 2013.

* CNRS (Centre Maurice Halbwachs). 48 Bd Jourdan, F-75014 Paris. caroline.guibetlafaye@ens.fr

* Caroline Guibet Lafaye est directrice de recherches au Centre Maurice Halbwachs (CNRS - EHESS - ENS) et a été Habilitée à Diriger des Recherches par l'Université de Paris-IV Sorbonne en 2011. Agrégée et docteur en philosophie de l'Université de Paris-I Panthéon-Sorbonne, elle consacre ses travaux à l'analyse de l'appréciation des inégalités et de leur légitimation, en contexte redistributif. Caroline Guibet Lafaye est l'auteur de plusieurs articles et ouvrages dans ces domaines, dont deux livres parus en 2006 aux Presses Universitaires de Laval : La justice comme composante de la vie bonne et Justice sociale et éthique individuelle, et un en 2012 : Le juste et l'inacceptable. Les sentiments d'injustice contemporains et leurs raisons (PUPS). Elle a également fait paraître en 2009 aux Presses Universitaires de Louvain un ouvrage sur : Penser le bonheur aujourd'hui. 
justice distributive. Une hypothèse explicative de cette incidence réside dans la cohérence intrinsèque des représentations morales - du juste -, cette cohérence signifiant corrélativement que les jugements normatifs individuels ne sont pas simplement l'expression d'intérêts sociaux, personnels, professionnels mais traduisent des convictions morales fondées et motivées par des principes.

Dans les conditions sociales actuelles de la société française, le positionnement politique s'avère plus explicatif des représentations du juste que la position sociale. Les préférences politiques traduisent et constitueraient un épiphénomène de logiques interprétatives générales et compréhensives plus fondamentales, structurant la compréhension du juste et la perception des inégalités. Dans ce qui suit, nous préciserons l'influence, dans la société française contemporaine, de la position sociale sur le jugement de justice et sur les sentiments d'injustice.

L'hétérogénéité des sentiments d'injustice, qui s'expriment selon les positions sociales individuelles, la variabilité des attitudes à l'égard des inégalités ou face au principe d'égalité suggèrent plusieurs pistes interprétatives et une élucidation des phénomènes d'acceptation des inégalités, de leurs mécanismes et des raisons qui les sous-tendent. Y a-t-il une identité sociale, politique et/ou d'autres traits communs chez les individus qui formulent des argumentaires d'acceptation ou, à l'inverse, de rejet des inégalités? Les positions sociales et les préférences politiques ont-elle une incidence sur la tolérance aux inégalités?

Pour envisager ces questions, nous nous appuierons sur l'enquête PISJ, dont le financement a été accordé par l'Institut de France (Fondation Simone et Cino Del Duca) à l'Académie des Sciences Morales et Politique et qui a été dirigée scientifiquement par O. Galland et M. Forsé. L'enquête comportait un volet quantitatif et un volet qualitatif. Le terrain de l'enquête quantitative s'est déroulé de septembre à octobre 2009 auprès d'un échantillon de 1711 individus représentatifs par quota de la population de 18 ans et plus, résidant en France métropolitaine. Le volet qualitatif de l'enquête PISJ, coordonné par l'auteure et Maxime Parodi, a été conduit, pour l'essentiel, durant l'année 2010 et le début de l'année 2011. Cinquante et un entretiens ont été réalisés dans cinq zones géographiques françaises: les régions de Grenoble, Lille, Lyon, Nantes, Paris. Ils ont été enregistrés et retranscrits intégralement pour ensuite être soumis à une analyse classique de contenu ainsi qu'à une analyse textuelle à l'aide du logiciel Alceste.

\section{Jugements de justice et positionnement politique}

\subsection{Normes utopiques et normes existentielles}

Le jugement de justice convoque des principes concernant la nature et l'origine desquelles la littérature demeure divisée: s'agit-il de principes abstraits de justice, universellement partagés ou bien de «normes existentielles», i.e. de normes effectivement adoptées et présidant à l'interaction sociale dans laquelle les acteurs sont immergés. Le modèle de la «valeur du statut» (status value), développé par Berger et al. (1972), suggère que les individus convoquent, dans leurs jugements et leurs attentes en matière de rétribution sociale, plutôt des normes existentielles que des normes utopiques ou des principes abstraits de justice. L'enquête sociologique par entretiens approfondis PISJ suggère de reconsidérer ces 
conclusions de la psychologie et de la sociologie expérimentales. Elle révèle, comme B. Wegener (1991) l'a également souligné, que les jugements de macrojustice sont guidés par des croyances politiques et idéologiques et tendent à être fondés sur des normes «utopiques» plutôt qu' «existentielles» ou «existantes» de justice (Jasso et Rossi, 1977).

La dénonciation, dans les récits recueillis, des défauts des distributions sociales actuelles ou des normes existentielles, orchestrant certains mécanismes sociaux ou politiques contemporains, se fonde de façon récurrente sur des normes idéales de justice. Ces dernières motivent la description de systèmes de régulation politique alternatifs où serait par exemple renforcé le contrôle démocratique ${ }^{1}$. Elles qualifient des sous-systèmes sociaux (l'éducation scolaire ou l'entreprise par exemple) ou les fonctionnements macrosociaux (l'économie, la fiscalité, les mécanismes d'embauche). Les propositions tendanciellement les plus innovantes et les moins marquées par l'existant sont, le plus souvent, formulées par des individus ayant des préférences politiques affirmées pour la gauche. Tel est également le cas de la désignation d'alternatives aux normes existentielles contemporaines. En revanche, les individus qui portent leurs votes vers la droite tendent à privilégier des mécanismes de régulation plus stricts dans les réformes qu'ils esquissent. Les normes «utopiques» consistent alors, pour certaines, dans la promotion de l'engagement individuel dans la solidarité sociale - en particulier chez des enquêtés ayant des préférences politiques pour la gauche, indépendamment de leur position dans l'espace social-, pour d'autres, dans la reconnaissance sociale réciproque des groupes sociaux, des professions et des fonctions aujourd'hui dévalorisées. Plusieurs enquêtés proposent des solutions originales susceptibles d'assurer davantage d'équité sur le marché du travail ou dans le monde de l'entreprise ${ }^{2}$.

Les entretiens menés confirment, pour certains enquêtés au profil très spécifique dont les positions politiques s'expriment, de façon marquée, en faveur de la gauche et de l'extrême gauche, la primauté de principes de justice fondamentaux dans les discours de dénonciation des injustices et des inégalités sociales, comme dans l'expression de sentiments d'injustice. Ainsi les individus privilégiant le vote à gauche défendraient des principes abstraits de justice, en un sens à la fois faible et fort, c'est-à-dire aussi bien des principes actuellement non implémentés mais aussi des principes (utopiques) appartenant à des conceptions compréhensives de la justice n'ayant pris forme dans aucune société. Les individus votant à droite soutiendraient, pour leur part, notamment dans certaines sphères de la justice particulières (comme le travail salarié ou la santé) des normes existantes telles que la loi de l'offre et de la demande, le principe de la rémunération des talents, celui de la réciprocité entre contribution et rétribution.

1 L'entretien avec Habib, conseiller principal d'éducation (CPE) de 56 ans, en offre un exemple: «heureusement qu'il y a des élections. Moi je dirais que le top, l'idéal étant donné que des gens sont mécontents en général, que ça soit par la droite ou par la gauche, le top c'est que personne ne vote! Au moins un mandat, et on verra bien qu'ils seront dans la merde... personne pourra gouverner, puisque personne n'aura été élu. Mais personne ne vote! C'est utopique, c'est utopique... Voilà. Mais ça ça reste pour moi un rêve de le voir un jour...».

2 Richard, cadre supérieur dirigeant de PME de 40 ans, évoque «des systèmes où tout le monde est actionnaire de sa société, il y a un vrai partage, des patrons qui plafonnent leur salaire parce qu'au-delà d'un certain salaire ils estiment que ça devient de la bêtise plus qu'autre chose». 


\subsection{Principe du mérite et orientations politiques}

Lorsque l'on envisage ce principe de justice distributive cardinal qu'est le mérite, l'analyse des données quantitatives de l'enquête PISJ (Guibet Lafaye, 2011a) suggère que les individus ne retrouvent pas, dans la réalité française contemporaine, les normes de justice décrivant le mérite personnel en termes d'efforts, de résultats, de talents, de diplômes, critères auxquels ils se montrent pourtant attachés. L'enquête PISJ, contrairement à des études précédemment menées (Miller, 1999; Piketty, 2003), montre que le principal clivage autour de l'interprétation du mérite - saisie dans la variable: «Des différences de revenu sont acceptables lorsqu'elles rémunèrent des mérites individuels différents» - ainsi que les raisons de la préférence axiologique pour ce principe ne sont pas tant d'ordre social que politique (Guibet Lafaye, 2011a, p. 133-134). Les individus se positionnant du côté gauche de l'échiquier politique récusent qu'il faille tenir compte des résultats et du diplôme pour fixer les niveaux de revenu. Les partisans de l'extrême gauche sont les plus sceptiques à l'égard de ce principe: ils mettent en doute sa légitimité aussi bien que ses critères, en l'occurrence les efforts, les résultats ou les talents. À l'inverse, les partisans de la droite traditionnelle formulent un attachement au principe du mérite, qu'ils interprètent de façon privilégiée en termes d'efforts et de talents plutôt qu'en termes de diplômes et de résultats.

En outre, une dichotomie des interprétations du mérite s'opère selon que les individus privilégient le vote à droite ou le vote à gauche. Ainsi l'expérience, entendue comme une compétence ou bien comme la connaissance de son métier, justifie, en particulier dans le discours des partisans de la gauche, une rémunération salariale supérieure. En revanche lorsqu'il est question des responsabilités de supérieurs hiérarchiques, ces enquêtés sont plus nombreux à prendre leurs distances à l'égard de l'idéologie dominante du mérite.

De la même façon, l'interprétation des principes de justice distributive et les conceptions du juste, en matière de distribution des ressources dans le champ de la santé publique, varie selon les orientations politiques. Du côté gauche de l'échiquier politique, les enquêtés privilégient une couverture universelle en santé, au nom de principes fondamentaux du type «Tout le monde doit être soigné pareil» (Marine). Une partie des partisans de la droite adhère également à cette logique universaliste mais une partie d'entre eux déploie un discours plus restrictif, indépendamment de leur situation sociale. Le système alternatif promu est de nature assurantiel. Il repose sur une norme de réciprocité entre contributions individuelles et bénéfices sociaux voire, chez les plus radicaux, sur la promotion de la responsabilité individuelle et de la responsabilisation des usagers de santé.

Ces exemples et récits confirment que les individus (quelle que soit leur position dans l'espace social mais plus particulièrement lorsqu'ils votent à gauche) nourrissent des attentes normatives à l'égard de la société dans laquelle ils vivent, précisément parce qu'ils ont une conception et une représentation abstraite de ce qu'est une société juste et une conception de celle-ci pour une large part forgée ex ante, contrairement à ce que la théorie sociale suggère souvent (Berger et al., 1972). Les sentiments d'injustice surgissent alors dans l'écart entre la représentation individuelle (éventuellement partagée collectivement) d'une société juste et ce que les individus perçoivent de l'état actuel de la société (Forsé et Parodi, 2009). 


\section{Conceptions de la justice, positions sociales et politiques}

\subsection{Facteurs sociaux vs. préférences politiques}

Le positionnement politique est en outre étroitement lié à et atteste de représentations du monde et d'interprétations caractérisées de la genèse des inégalités. Selon les représentations morales et les conceptions de l'ordre social endossées (s'ordonnant par exemple en référence au libre marché, à la lutte des classes ou à des formes d'exploitation entre groupes sociaux), les individus déploient des interprétations hétérogènes de la genèse des inégalités et de leur légitimité. La préférence pour telle ou telle norme (égalité, besoin ou mérite) dans les jugements de justice est indissociable de la légitimité qui lui est reconnue dans un contexte donné, cette légitimité étant conditionnée par des représentations sociales et morales, par des visions du monde plus générales et par des conceptions de la responsabilité (Kellerhals, 2003, p. 140).

La prise en compte de l'auto-positionnement politique, qui traduit dans le champ public et politique, la préférence individuelle pour telle représentation du monde plutôt que pour telle autre, serait aujourd'hui un facteur explicatif prévalent, tendant à relativiser «l'effet position sociale» qui dispose les individus les plus avantagés socialement à privilégier une explication individualiste - plutôt que structurelle - de la genèse des inégalités. L'hypothèse de la domination sociale, stipulant que les groupes ne font pas les mêmes expériences selon leurs positions dans la hiérarchie sociale, interprétées en termes d'oppression et de domination (Sherif, 1967), semble ainsi s'estomper au profit d'autres paradigmes interprétatifs, fondés sur la cohérence des convictions morales et politiques, comme Kreidl (2000) l'a également constaté.

Des analyses, tenant compte du raisonnement moral et des attitudes politiques, ont montré que le niveau «conventionnel» de Kohlberg (1981), et plus spécifiquement le stade 4, où se déploie le raisonnement moral conventionnel, est généralement associé au conservatisme et à une idéologie politique de droite (Fishkin, Keniston et Mac Kinnon, 1973; Rest et al., 1974; Candee, 1976; Krebs, Vermeulen, Carpendale et Denton, 1991), tandis que les orientations libérales - au sens anglo-saxon - sont positivement corrélées au stade 5 de type «postconventionnel». Le raisonnement moral conventionnel présente également des convergences avec certaines opinions sociales traditionnellement conservatrices, souvent de type répressive (comme la peine capitale (de Vries et Walker, 1986) ou associées à l'encouragement de solutions militaires (Westman et Levandowski, 1991). Ces corrélations émergent également dans des contextes théoriques non kohlbergiens où l'on a montré que les sujets ayant tendance à endosser l'éthique de la conscience personnelle (Hogan, 1973) se caractérisent par des orientations politiques libérales, tandis que ceux adoptant préférentiellement l'éthique de la responsabilité sociale sont plus conservateurs (Lorr et Zea, 1977; Gutkin et Suls, 1979)3.

La convergence entre représentations du monde, principes explicatifs des états du monde et options politiques se vérifie spécifiquement sur les questions de justice sociale. L'explication d'un état du monde par la croyance en un monde juste, la croyance en un monde

3 Une interprétation possible de ces corrélations consiste à associer le conservatisme social à un style cognitif et plus généralement certains fonctionnements cognitifs (Stone, 1986) à certaines idéologies. 
injuste ou la croyance en un monde aux événements aléatoires est étroitement dépendante des croyances et des convictions politiques. La mobilisation de la croyance en la justice du monde est corrélée au conservatisme (Rubin et Peplau, 1975; Wagstaff et Quirk, 1983) ainsi qu'à certaines attitudes sociales, tendant par exemple à dévaloriser les personnes victimes d'injustices sociales, en l'occurrence les femmes ou les Noirs (Rubin et Peplau, 1973), ou bien à imputer la pauvreté à une responsabilité personnelle (Furnham et Gunter, 1984).

\subsection{Croyances en la volonté individuelle: mobilité sociale et positions politiques}

Les croyances et opinions concernant le mode de génération des inégalités se nourrissent d'informations saisies au cours des parcours individuels. Avec la connaissance par ouï-dire, la trajectoire personnelle constitue l'une des sources majeures d'information à laquelle les individus puisent les matériaux de leur réflexion et de leurs interprétations dans le domaine. Les différences de trajectoires sociales expliquent une part de la variabilité des interprétations de la genèse des inégalités. Les positions sociales et les trajectoires individuelles (de mobilité sociale ascendante ou descendante) disposent les individus à privilégier certains principes axiologiques ainsi qu'à recourir à telle ou telle norme de justice dans leurs évaluations morales. Cependant et contrairement aux conclusions des théories de la domination sociale, le niveau de diplôme ainsi que le positionnement politique semblent davantage expliquer, dans les entretiens menés PISJ, l'élaboration abstraite des conceptions de la justice que les positions au sein de l'espace social ou professionnel.

La socialisation primaire et la socialisation secondaire ont également un effet sur les représentations que les personnes se font des inégalités et de leur genèse. Lorsque l'on considère l'expérience de la pénibilité du travail, on observe qu'elle pèse de façon différenciée sur les représentations morales individuelles. La pénibilité personnellement vécue paraît plutôt induire des dispositions individualistes, conformément à la logique: «j’y suis arrivé, donc on peut y arriver». Tel est moins le cas lorsque l'on est témoin de cette pénibilité, dans une expérience de niveau $\mathrm{N}+1$ dans son cadre familial par exemple et que, grâce à des études, on est parvenu soi-même à accéder à des métiers moins durs physiquement ou bien lorsque l'on se trouve être un témoin éloigné de cette pénibilité. En revanche, chez des individus portant leur vote à droite, quelle que soit leur position dans l'espace social, la prise en compte $\mathrm{du}$ vouloir individuel et des efforts personnels a un rôle décisif dans les jugements. Ainsi c'est en référence à la dichotomie responsabilité sociale vs. responsabilité individuelle que les personnes tirent des conclusions d'expériences de confrontation aux inégalités faites, en particulier, dans ce lieu d'apprentissage social privilégié qu'est le monde du travail.

En matière de justification des inégalités et des différentiels de positions sociales, le sentiment que «si l'on veut, on peut» joue un rôle avéré, dans la pensée profane (Staerklé et al., 2007, p. 249). Néanmoins la légitimité donnée au volontarisme ne semble aujourd'hui pas tant liée au niveau de ressources culturelles ou économiques - comme l'avait souligné Hochschild (1981) - qu'aux préférences politiques et à la spécificité des expériences personnelles. Ainsi la croyance au pouvoir de la volonté individuelle s'avère être une disposition très fortement assumée par des professions indépendantes, par des personnes ayant connu des trajectoires sociales favorables ou avouant des positions très individualistes (comme Pascal, ouvrier qualifié intérimaire de 38 ans qui reconnaît avoir un comportement de passager clandestin (free 
rider) à l'égard de l'assurance chômage). La référence à leur propre trajectoire est prégnante - et souvent explicite - de la part d'enquêtés faiblement leur diplômés. Pour ces derniers, le parcours personnel, en particulier lorsqu'il témoigne de formes de succès personnel, constitue la source principale d'informations servant à valider leurs jugements normatifs. Les personnes les plus favorisées, lorsqu'elles ont bénéficié d'une forte ascension sociale, font un usage plus nuancé de cette référence autocentrée et tendent moins à s'instituer en exemples. Y font exception celles développant une image de soi comme être autonome. Alors qu'on ne peut plus aujourd'hui conclure que les catégories supérieures pensent que les inégalités fondées sur l'effort et le talent sont légitimes (Dubet, 2006, p. 272), en revanche des trajectoires personnelles favorables nourrissent, chez leurs bénéficiaires, des croyances relatives au pouvoir de la volonté sur les destins personnels, quelles que soient leurs positions dans l'espace social.

La mobilité sociale semble donc avoir un effet majeur sur l'appréciation, à court et à moyen termes, des inégalités sociales, c'est-à-dire sur la façon dont les individus collectent et traitent l'information à laquelle ils sont confrontés comme acteurs sociaux. On ne peut donc plus si simplement conclure, comme le suggéraient les approches sociologiques fondées sur l'intérêt personnel, que l'explication de l'inégalité et de la pauvreté par l'individualisme croît avec l'élévation de la position sociale. L'analyse des entretiens PISJ souligne plutôt le rôle prépondérant du positionnement politique sur celui du niveau de revenu.

En effet, les orientations politiques personnelles introduisent des variations notables eu égard à l'incidence de la mobilité sociale: tous les individus qui ont bénéficié d'une mobilité sociale ascendante n'attribuent pas systématiquement leur succès à leurs efforts et à leurs compétences, contrairement à ce que suggèrent Kluegel et Smith (1986). Cette interprétation est assumée diversement selon que les enquêtés avouent une préférence pour la gauche ou la droite de l'échiquier politique. Le positionnement politique fait obstacle, dans certains cas, à la croyance en un monde juste (Lerner, 1980), c'est-à-dire à la conviction que chacun mérite son statut social et est responsable de ses résultats, ou encore à une explication individualiste des inégalités sociales. La foi dans le volontarisme est atténuée chez les enquêtés les plus favorisés socialement, votant à gauche, ceux-ci se révélant conscients des phénomènes de déterminisme social et d'inégalité des dotations individuelles face à l'effort. Toutes les personnes, fortement dotées socialement et culturellement, ne se déclarent donc pas ni ne se veulent maîtresses de leur destin, en référence à une image de soi, fondée sur la valeur de l'être autonome contrairement à ce que la littérature a pu, par le passé, conclure (Kellerhals, 1974, p. 145). Les orientations politiques et les parcours personnels jouent un rôle discriminant dans l'émergence de ces représentations. Non seulement la référence au libre choix traverse l'ensemble des récits mais la construction de soi comme individu autonome et maître de son destin ne constitue pas le dénominateur commun des représentations morales des individus interrogés les plus favorisés.

\section{Sentiments d'injustice, situations sociales et préférences politiques}

\subsection{Une sensibilité politique aux inégalités}

Ainsi les jugements de justice et les principes normatifs mobilisés varient selon les orientations politiques. Cette hétérogénéité affecte également les sentiments d'injustice qui 
s'expriment différemment selon les milieux sociaux et, en particulier, selon les positions sociales mais aussi selon les préférences politiques. L'enquête par questionnaire PISJ suggère que les représentations des inégalités sociales dans la société française sont hétérogènes au sein des catégories socioprofessionnelles (Guibet Lafaye, 2011b). De même, les attitudes normatives à l'égard des inégalités, se déployant dans des discours de légitimation ou de critique sociale, varient selon les professions. Les dénonciations peuvent cibler des inégalités spécifiques comme le montrent les jugements des cadres supérieurs en matière d'accès aux soins de santé (voir tableau 1). Le rejet des inégalités peut traduire une attitude générale face à toute différence comme le suggèrent les jugements des professions intermédiaires qui présentent une aversion généralisée aux inégalités. Dans d'autres configurations, la position personnelle pèse sur les jugements normatifs: les cadres supérieurs se révèlent plus tolérants que les autres catégories socioprofessionnelles aux inégalités de revenus et de patrimoine, alors que les professions intermédiaires et les ouvriers/employés, plus touchés par celles-là, se montrent plus sensibles aux inégalités de logement (voir tableau 1) $)^{4}$.

Cependant la distribution socioprofessionnelle des sentiments d'injustice est fortement concurrencée par l'influence des préférences politiques. Se dessine une congruence des options politiques et des représentations morales. Plus que la position sociale, le facteur décisif en matière de sensibilité aux inégalités sociales semble être le positionnement politique ainsi que le suggèrent les discours et jugements recueillis des individus portant leur vote à droite, indépendamment de leur position sociale. De façon analogue, les réponses à la question de l'existence de différents types d'inégalités en France, dans l'enquête par questionnaire, manifestent des écarts à la moyenne très marqués pour les individus déclarant voter à gauche et ceux déclarant voter à droite (voir tableau 2). Les premiers tendent à surestimer l'existence des inégalités en France relativement aux seconds, si l'on excepte les jugements portés sur les inégalités face au chômage, aux risques technologiques, à l'insécurité et aux générations (sachant que les personnes plus âgées ont davantage tendance à voter à droite). Plus fondamentalement, l'inacceptabilité des inégalités, toutes catégories confondues ${ }^{5}$, est systématiquement toujours plus prononcée parmi les individus affirmant des préférences politiques pour la gauche plutôt que pour la droite (tableau 3). Quand bien même les individus votant à gauche n'auraient pas toujours autant conscience de la prégnance de certaines inégalités, ils les dénonceront systématiquement, révélant par là une position tranchée à leur égard. À l'inverse, la préférence politique pour la droite est corrélée avec un degré supérieur de tolérance aux inégalités aussi bien d'un point de vue normatif qu'en matière de sensibilité à ces dernières, indépendamment du domaine dans lequel elles sévissent effectivement.

Ainsi la prise en compte des opinions politiques, en matière de perception et de tolérance aux inégalités, laisse surgir des écarts plus notables que lorsque sont envisagés les niveaux

4 Telle est également la position normative des artisans mais pour d'autres raisons. Les agriculteurs moins exposés à ces difficultés s'y montrent relativement plus tolérants.

5 C'est-à-dire «les inégalités de revenu, de patrimoine, les inégalités face au chômage et aux emplois précaires, les inégalités dans les études scolaires, les inégalités d'accès aux soins médicaux, d'exposition aux risques technologiques, industriels ou scientifiques (nucléaire, OGM, santé...), les inégalités liées à l'origine ethnique, les inégalités entre les hommes et les femmes, entre les jeunes et les plus âgés, face à l'insécurité, les inégalités de logement, les inégalités de pénibilité du travail». 
de revenu. La comparaison des $\mathrm{V}$ de Cramer montre que les opinions politiques expliquent davantage l'appréciation de la réalité des inégalités que la catégorie socioprofessionnelle ou le niveau de revenu (tableau 4). Tel est également le cas pour la tolérance aux inégalités, si ce n'est pour les inégalités de patrimoine, où le niveau de revenu pèse davantage, et pour l'acceptabilité des inégalités intergénérationnelles où le facteur socioprofessionnel est plus déterminant. Se dessine ainsi une nette corrélation entre sentiments d'injustice et positionnement politique. L'analyse des discours de la phase qualitative de l'enquête PISJ permet en outre de saisir la très forte convergence et le recouvrement entre l'idéologie politique contemporaine et le discours sur les inégalités, leur émergence et les mécanismes interprétatifs du fonctionnement social.

\subsection{Sentiments d'injustice typiques et figures de l'altérité}

D'une part, le positionnement politique - traduisant des convictions morales - n'a pas simplement un effet sur la substance des sentiments d'injustice et leur intensité mais également sur leur intentionnalité. Une double causalité se dessine conjuguant les préférences politiques et le positionnement au sein de l'espace social. En effet, le sens et la portée conférés aux sentiments d'injustice dépendent de l'acteur concerné: le locuteur $(\mathrm{N}+0)$, les proches $(\mathrm{N}+1)$, les autres (tels les passagers clandestins) $(\mathrm{N}+\mathrm{n})$, la société, les sentiments d'injustice étant, dans ce cas, provoqués par le fonctionnement macrosocial $(\mathrm{N}+\infty)$. Les personnes privilégiant le vote à droite exprimeront davantage des sentiments d'injustice à l'endroit des «autres» $(\mathrm{N}+\mathrm{n})$, en l'occurrence des «autres» non vertueux que sont par exemple les passagers clandestins. De même, les individus ayant des positions socialement privilégiées formuleront davantage des jugements qui mettent en question autrui et endossent une attitude moins compréhensive à l'égard des plus favorisés. À l'inverse les personnes ayant des situations sociales et professionnelles moins avantagées tendront davantage à mettre en question le système ou la structure $(\mathrm{N}+\infty)$. Cette dernière attitude critique se trouve renforcée quand ces individus affirment des préférences politiques pour la gauche de l'échiquier politique. En effet, les individus déclarant voter à gauche, quelle que soit leur position dans l'espace social, se montreront plus sensibles aux injustices du fonctionnement macrosocial $(\mathrm{N}+\infty)$ ainsi qu'à celles émergeant de contextes microsociaux et dont ils ont pu être témoins $(\mathrm{N}+2)$. Cette dernière occurrence se vérifie en particulier dans le monde professionnel. Le vote à gauche, dans les espaces sociaux avantagés, semble donc être un meilleur prédictif de la nature des sentiments d'injustice incriminant prioritairement la structure sociale pour les situations d'inégalités et d'injustice considérées. Le vote à gauche et les positions sociales faiblement avantagées coïncident en outre souvent avec une empathie plus forte à l'égard des individus en situation difficile et défavorisés par les inégalités évoquées.

Ainsi le positionnement politique - à gauche comme à droite - paraît fortement lié à l'expression de sentiments d'injustice typiques ainsi qu'à une préoccupation pour des niveaux différenciés auxquels des injustices surgissent. Le souci empathique pour les inégalités sociales induisant des torts envers des groupes sociaux désignables est plus important à gauche. Ne se trouve toutefois alors soulignée qu'une cohérence des conceptions morales puisque cette attention sociale définit intrinsèquement et explique la singularité de ce positionnement politique. Se dessine donc moins une causalité entre ces deux dimensions qu'un 
rapport réciproque puisque le positionnement politique condense et traduit un ensemble de sentiments d'injustice et de convictions morales relatifs à des états du monde contemporains.

Pour une autre part, la figure de «l'autre» s'esquisse de façon distincte dans les jugements selon les positions de ceux qui se prononcent dans l'espace social et selon les préférences de vote. Du côté socialement avantagé et lorsque les individus votent à droite, «les autres», ce sont, de façon récurrente, les pauvres non méritants, les bénéficiaires de revenus excessifs, en particulier les footballeurs, alors qu'à l'autre extrémité de l'échelle sociale partageant ce positionnement politique, «les autres» ont plus souvent la figure du patronat ou des fainéants. En ce sens, l'absence de ressources ne constitue pas systématiquement une caution pour l'ostracisme fondant ses critères de juste sanction sur une image statutaire ou catégorielle du criminel (souvent appréhendé comme l'étranger), du malade mental ou du drogué, systématiquement considérés comme autres (Kellerhals, 1974, p. 145). Dans la désignation de ces «autres», qui subissent l'injustice ou qui la perpétuent, s'esquisse la constitution d'une altérité dont la fonction déborde celle à laquelle les théories de l'équité l'ont assignée, lorsqu'elles jugeaient de l'évaluation de l'équité de leur rémunération par les individus (Berger et al., 1972; Cook, 1975; Jasso, 1980). L'instanciation d'une altérité traverse les jugements normatifs de justice en général plutôt que simplement les évaluations de sa propre rémunération. Se dessine, par exemple, une classe de sentiments d'injustice dénonçant une asymétrie favorable à ces «autres» puissants (et souvent non vertueux) que sont les patrons, les puissants, les hommes politiques.

La dénonciation de ces «autres» non vertueux - qui nourrit des sentiments d'injustice typiques $-\mathrm{s}$ 'articule à des principes de justice spécifiques. Un sentiment d'injustice caractéristique, opérant dans le discours des individus privilégiant le vote à droite, stigmatise l'inégale répartition des coûts sociaux et de la contribution sociale, dans une pluralité de sphères de la justice aussi bien que concernant des comportements de passager clandestin, associés ou non à une désapprobation morale ${ }^{6}$.

Alors que l'équité de la contribution sociale constitue un principe structurant des conceptions de la justice, indépendant des positions sociales et politiques des individus, en revanche, la référence à la paresse joue le rôle de caution morale dans un discours qui dénonce le free riding. Sur cette question, le partage idéologique majeur s'opère entre individus votant à droite et individus votant à gauche. Ainsi concernant l'interprétation des causes de la pauvreté, une nette opposition se noue, au plan statistique, selon que les individus manifestent des préférences politiques en faveur de la droite ou de la gauche de l'échiquier politique (voir Guibet Lafaye, 2011b, tableau 7.4.1). Alors que la droite impute majoritairement la pauvreté à la paresse ou à la mauvaise volonté (et, pour partie d'entre elle, à l'absence de soutien familial), la gauche privilégiera nettement l'explication structurelle à partir des contraintes inhérentes au marché de l'emploi ou en référence à la malchance (voir Guibet Lafaye, 2011b, tableaux 7.4.1 à 7.4.3). Lorsque l'on tient compte du niveau de diplôme et des préférences politiques, il apparaît que les individus les moins diplômés votant à droite expliquent la pauvreté par la paresse à la différence de ceux votant à gauche

6 Jean-Baptiste, cadre supérieur du privé de 44 ans, considère que «ce qui n'est pas acceptable, c'est quelqu'un à qui on propose quinze fois un job et qui dit non parce que juste il n'a pas envie de travailler. Quelqu'un qui pourrait bien faire le job et qui le refuse parce qu'il n'a pas envie de travailler, ce n'est pas normal...». 
(voir Guibet Lafaye, 2011b, tableaux 7.5.1 à 7.5.3). Une opposition interprétative similaire se retrouve parmi les individus les plus diplômés du supérieur. Ce partage explicatif entre la gauche et la droite traverse toutes les catégories de diplômes et montre ainsi que cette interprétation de la genèse de la pauvreté est structurante des représentations qu'en ont les individus votant à droite.

Le vote traduit donc une conception du monde et une représentation morale, et constitue ainsi un prédictif de positions morales en matière de justice sociale. Les enquêtés qui votent à droite auront davantage le sentiment qu'ils travaillent et paient des impôts alors que d'autres restent tranquillement chez eux à profiter des aides sociales sans travailler et vivent des bénéfices dégagés par les premiers. Ce sentiment est rémanent chez ces partisans politiques indépendamment de leur position dans l'espace social.

À l'inverse, l'attitude des personnes, socialement favorisées, votant à gauche consiste plutôt à dénoncer les comportements stigmatisants des plus favorisés à l'égard des moins favorisés. À certaines occasions, les discours des individus se situant sur la gauche de l'échiquier politique dénoncent le cumul d'avantages associés à une situation d'abus par rapport à des individus moins favorisés ${ }^{7}$. Le sentiment d'injustice est nourri par la double exclusion suscitée par le cumul des avantages et affectant ceux - et celles, en l'occurrence - qui n'en bénéficient pas ou par le cumul des coûts sociaux, portant sur les catégories les plus défavorisées de la population.

De même, les sentiments d'injustice suscités par la conscience de l'exploitation, induite par des situations de domination sociale, sont récurrents et plus affirmés à gauche. Le sentiment d'instrumentalité et d'exploitation - dénonçant par lui-même un jeu biaisé de l'interaction sociale (Boudon, 1995) - nourrit une représentation de cette dernière fondée sur «l'effet de sablier», c'est-à-dire sur la conviction que si certains gagnent au jeu social c'est parce que d'autres sont relégués dans des positions de perdants. Jorou, professeur des écoles de 40 ans, en donne une formulation paradigmatique: «J'ai tendance à penser que les gens qui gagnent énormément en général ça leur est permis au détriment des autres. La richesse se construit sur le dos des autres et pour devenir riche il faut absolument exploiter les autres».

\section{Conclusion}

La détermination par l'empirique et l'existentiel des jugements de justice ne tient pas seulement aux inégalités envisagées ou aux configurations socioéconomiques à travers lesquelles elles se dessinent. Les positions sociales, les préférences politiques et les expériences vécues de l'injustice influencent les jugements de justice. Cependant la sociologie empirique permet de mettre en évidence une autonomie relative des représentations du juste, c'est-àdire des raisonnements concernant la micro- et la macrojustice. Elle se traduit notamment par une sensibilité morale caractérisée, chez certains individus, ou dans la préférence marquée pour certains principes de justice qui porteront les personnes à éprouver, face à certaines situations, des sentiments d'indignation que d'autres ignoreront. Cette sensibilité s'exprime

7 Jannick, ouvrier qualifié de 57 ans, commente également la négociation de la réforme des retraites en France en 2010: «Le projet de loi n'est pas équitable dans son ensemble par rapport à ce que le gouvernement propose: que ce soit uniquement les travailleurs qui paient cette retraite à 62 ans et que l'assiette des recettes ne soit pas élargie aux revenus financiers». 
dans une valorisation de l'humain et de son traitement respectueux, dans un souci de l'équité de traitement individuel, signe d'une égale dignité reconnue à chacun. Elle sous-tend également une morale attentive et empathique à l'égard des plus défavorisés, se traduit dans des principes de justice distributive vecteurs d'une équité sociale plus réelle dans un souci d'une contribution sociale accrue de tous, dans le non accaparement des bénéfices de l'interaction sociale par certains, dans la récusation de la reproduction sociale et de la perpétuation de l'inégalité des chances. Cette sensibilité s'exprime fortement du côté gauche de l'échiquier politique ${ }^{8}$. Ainsi cette sensibilité morale plutôt que les expériences d'injustice, personnellement vécues, pourrait être le facteur explicatif principal des jugements et des sentiments d'injustice ainsi que de certaines orientations politiques. La place centrale conférée à certains principes de justice fondamentaux, dans la dénonciation ou l'expression de sentiments d'injustice en atteste, tout de même que l'attitude à l'égard des inégalités et les jugements d'injustice d'enquêtés exprimant des préférences politiques marquées en faveur de la gauche et de l'extrême gauche.

Cette sensibilité morale motive des sentiments d'injustice pour des situations ne concernant pas personnellement les individus s'exprimant. Alors qu'on ne peut systématiquement affirmer que le sentiment d'injustice est d'autant plus intense que l'individu est intimement concerné par la situation qu'il dénonce, cette intensité est en revanche toujours d'autant plus forte que les préférences politiques individuelles se portent vers la gauche de l'échiquier politique, si ce n'est, lorsque les individus affirmant une préférence pour la droite, dénoncent les comportements de passager clandestin.

La prévalence explicative du positionnement politique concernant la perception des inégalités sur l'effet «position sociale», aussi bien dans les données quantitatives que qualitatives, témoigne d'une incontestable cohérence des représentations morales. D'une part, la distribution des sentiments d'injustice semble plus fortement affectée par les préférences politiques que par les positions sociales dans les deux approches méthodologiques convoquées. D'autre part, l'inacceptabilité de catégories d'inégalités, systématiquement répertoriées, est en général plus affirmée parmi les partisans de la gauche. La proximité politique avec la droite est corrélée à un degré supérieur de tolérance aux inégalités aussi bien d'un point de vue normatif qu'en matière de sensibilité à la réalité de ces dernières, hormis pour certains types d'inégalités dont on peut penser qu'ils concernent davantage les individus ayant ces préférences. La corrélation très nette entre sentiments d'injustice et positionnement politique suggère une cohérence des représentations, articulant interprétation de la genèse des inégalités, appréciations normatives de ces dernières et mécanismes interprétatifs du fonctionnement social. Les préférences politiques, traduisent dans le champ public et politique, des convictions individuelles relatives à l'émergence de phénomènes macrosociaux et coïncident avec des représentations du monde spécifiques. La corrélation fondamentale des préférences politiques et des jugements de justice atteste de la cohérence des logiques interprétatives et des représentations morales, structurant la compréhension du juste et la perception des inégalités.

8 Nous avons noté que du côté droit, le souci du pragmatisme, des contraintes économiques et de l'efficacité est plus prégnant. S'y associent la croyance dans le volontarisme, le souci de la rémunération des talents ainsi que l'attention à la réciprocité entre rétribution et contribution. 


\section{Références}

J. BERGER, M. JR ZELDITCH, B. ANDERSON, B.P. COHEN: «A Status Value Formulation», en: J. Berger, M. Jr Zelditch et B. Anderson (eds.): Sociological Theories in Practice, t. 1, Boston, Houghton-Mifflin, 1972, pp. 119-146.

R. BOUDON: Le juste et le vrai, Paris, Fayard, 1995.

D. CANDEE: «Structure and choice in moral reasoning», Journal of Personality and Social Psychology, vol. 34, 1976, pp. 1293-1301.

KAREN S. COOK: «Expectations, Evaluations and Equity», American Sociological Review, vol. 40, n 3, 1975, pp. 372-388.

B. DE VRIES et L. WALKER: «Moral reasoning and attitudes towards capital punishment», Developmental Psychology, vol. 22, $\mathrm{n}^{\circ}$ 4, 1986, pp. 509-513.

F. DUBET: Injustices. L'expérience des inégalités au travail, Paris, Seuil, 2006.

J. FISHKIN, K. KENISTON et C. MCKINNON: «Moral reasoning and political ideology», Journal of Personality and Social Psychology, vol. 27, n 1, 1973, pp. 109-119.

M. FORSÉ et M. PARODI: «Une théorie de la cohésion sociale», La revue Tocqueville, vol. XXX, n 2, 2009, pp. 9-35.

A. FURNHAM et B. GUNTER: «Just World belief and attitudes toward the poor», British Journal of Social Psychology, 23, 1984, pp. 265-269.

CAROLINE GUIBET LAFAYE: «Le mérite et ses dimensions», en: M. Forsé et O. Galland (eds.): Les Français face aux inégalités et à la justice sociale, Paris, Colin, 2011a, pp. 128-138.

CAROLINE GUIBET LAFAYE: «Le jugement de justice et ses raisons. Le juste et l'inacceptable», mémoire d'Habilitation à Diriger des Recherches, Université Paris Sorbonne, $2011 b$.

CAROLINE GUIBET LAFAYE: Le juste et l'inacceptable. Les sentiments d'injustice contemporains et leurs raisons, Paris, PUPS, 2012.

D.C. GUTKIN, J. SULS: «The relation between the ethics of personal conscience-social responsibility and principled moral reasoning», Journal of Youth and Adolescence, vol. 8, n 4, 1979, pp. 433-441.

J. HOCHSCHILD: What's Fair? American Beliefs about Distributive Justice, Cambridge Mass., Harvard University Press, 1981.

R. HOGAN: «Moral conduct and moral character: A psychological perspective», Psychological Bulletin, vol. 79, $\mathrm{n}^{\circ}$ 4, 1973, pp. 217-232.

GUILLERMINA JASSO et PETER H. ROSSI: «Distributive Justice and Earned Income», American Sociological Review, vol. 42, 1977, pp. 639-651.

G. JASSO: «A new theory of distributive justice», American Sociological Review, vol. 45, 1980, pp. 3-32.

JEAN KELLERHALS: Les associations dans l'enjeu démocratique, Lausanne, Payot, 1974.

JEAN KELLERHALS: «Relativisme et sociologie: le cas du sentiment de justice», Revue européenne des sciences sociales, $\mathrm{n}^{\circ}$ XLI-126: Sociologie et relativisme, 2003, pp. 137-150.

KLUEGEL J. et E. SMITH: Beliefs about inequality. Americans' view of what is and what ought to be, New York, Aldine De Gruyter, 1986. 
L. KOHLBERG: Essays on moral development, vol. 1: The philosophy of moral development, New York, Harper \& Row, 1981.

D.L. KREBS, S.C. VERMEULEN, J.I. CARPENDALE, K. DENTON: «Structural and situational influences on moral judgment: The interaction between stage and dilemmas», en: W.M. Kurtines et J.L. Gewirtz (eds.): Handbook of moral behavior and development, t. 1, Hillsdale, Lawrence Erlbaum, 1991, pp. 139-169.

MARTIN KREIDL: «What makes inequalities legitimate? An international comparison», 2000, research paper $\mathrm{n}^{\circ}$ 403/99/1128, ISBN 80-85950-80-4.

M.J. LERNER: The belief in a just world: A fundamental delusion, New-York, Plenum Press, 1980.

M. LORR et R.L. ZEA: «Moral judgment and liberal-conservative attitude», Psychological Reports, vol. 40, 1977, pp. 627-629.

D. MILLER: Principles of Social Justice, Cambridge (Mass.) et Londres, Harvard University Press, 1999.

T. PIKETTY: «Attitudes vis-à-vis des inégalités de revenu en France», Comprendre, $\mathrm{n}^{\circ} 4$, 2003, pp. 224-227).

J.R. REST, D. COOPER, R. CODER, J. MANSANZ, D. ANDERSON: «Judging the important issues in moral dilemmas. An objective measure of development», Developmental Psychology, vol. 10, 1974, pp. 491-501.

Z. RUBIN et L.A. PEPLAU: «Who believes in a just world?», Journal of Social Issues, vol. 31, n 3, 1975, pp. 65-89.

SHERIF M., Group conflict and cooperation: Their Social Psychology, Londres, Routledge et Kegan Paul, 1967.

C. STAERKLÉ, C. DELAY, L. GIONETTONI et P. ROUX, Qui a droit à quoi? Représentations et légitimation de l'ordre social, Grenoble, Presses universitaires de Grenoble, 2007.

W.F. STONE: «Personality and ideology: Empirical support for Tomkin's polarity theory», Political Psychology, vol. 7, n 4, 1986, pp. 689-706.

G.F. WAGSTAFF et M.A. QUIRK: «Attitudes to sex roles, political conservatism and belief in the just world», Psychological Reports, vol. 2, 1983, pp. 151-176.

WEGENER BERND: «Relative deprivation ans social mobility: structural constraints on distributive justice judgments», European Sociological Review, vol. 7, n 1, 1991, pp. 3-18.

A.S. WESTMAN et L.M. LEWANDOWSKI: «How empathy, egocentrism, Kohlberg's moral development, and Erikson's psychosocial development are related to attitudes toward war», Psychological Reports, vol. 69, 1991, pp. 1123-1127. 


\section{Annexes}

Tableau 1

Perception de la réalité et de l'acceptabilité des inégalités selon les catégories socioprofessionnelles

\begin{tabular}{|c|c|c|c|c|c|c|c|c|c|c|}
\hline & \multicolumn{2}{|c|}{ Agriculteurs } & \multicolumn{2}{|c|}{ Artisans } & \multicolumn{2}{|c|}{ Cadres sup } & \multicolumn{2}{|c|}{ Prof inter } & \multicolumn{2}{|c|}{ Ouv \& employés } \\
\hline & Fortes & Acceptables & Fortes & Acceptables & Fortes & Acceptables & Fortes & Acceptables & Fortes & Acceptables \\
\hline Les inégalités de revenu & 7,45 & 5,08 & 7,54 & 4,10 & 8,00 & 4,77 & 8,15 & 4.26 & 7,88 & 3,84 \\
\hline Les inégalités de patrimoine & 6,48 & 5,39 & 6,86 & 4,87 & 7.67 & 5,27 & 7,42 & 5,10 & 7,08 & 4,59 \\
\hline $\begin{array}{l}\text { Les inégalités face au chômage et aux emplois } \\
\text { précaires }\end{array}$ & 6,48 & 4,14 & 7,14 & 3,57 & 7,45 & 3,68 & 7,38 & 3,43 & 7,47 & 3,43 \\
\hline Les inégalités dans les études scolaires & 5,88 & 3,96 & 5,94 & 3,71 & 6,50 & 3,67 & 6,72 & 3,49 & 6.66 & 3,63 \\
\hline Les inégalités d'accès aux soins médicaux & 5,24 & 3,77 & 6,18 & 3,12 & 5,71 & 3,03 & 6,40 & 2,88 & 6,53 & 3,09 \\
\hline Les inégalités liées à l'origine ethnique & 6,13 & 4,48 & 6,31 & 2,86 & 7,17 & 2,97 & 7,20 & 3,07 & 6,87 & 3,35 \\
\hline Les inégalités entre les hommes et les femmes & 5,81 & 3,55 & 5,90 & 3,00 & 6,62 & 3,15 & 6,59 & 2,94 & 6,64 & 3,21 \\
\hline Les inégalités entre les jeunes et les plus âgés & 5,18 & 3.70 & 6.14 & 3,78 & 6.36 & 3.75 & 6,29 & 3,49 & 6.36 & 3.61 \\
\hline Les inégalités de logement & 6,20 & 3,79 & 7.23 & 3,07 & 7,41 & 3.26 & 7.49 & 3,13 & 7,46 & 3,15 \\
\hline Les inégalités de pénibilité du travail & 6.68 & 3.97 & 7.15 & 3.35 & 7,37 & 3,62 & 7,59 & 3.50 & 7.54 & 3.37 \\
\hline
\end{tabular}

Moyenne des réponses aux questions: (a) «Il existe plusieurs types d'inégalités dans la société française. Pour celles que je vais vous citer dites-moi si selon vous elles sont fortes ou pas aujourd'hui? Donnez votre réponse sur une échelle allant de 1 pour «Pas fortes du tout» à 10 pour «Très fortes»; (b) «Pour chacune des inégalités suivantes, quelles sont celles qui vous semblent plus ou moins acceptables? Donnez chaque fois votre réponse sur une échelle allant de 1 pour «ne sont jamais acceptables» à 10 pour «sont toujours acceptables».

\section{Tableau 2}

\section{Appréciation de la force des inégalités en France selon les préférences politiques ${ }^{9}$}

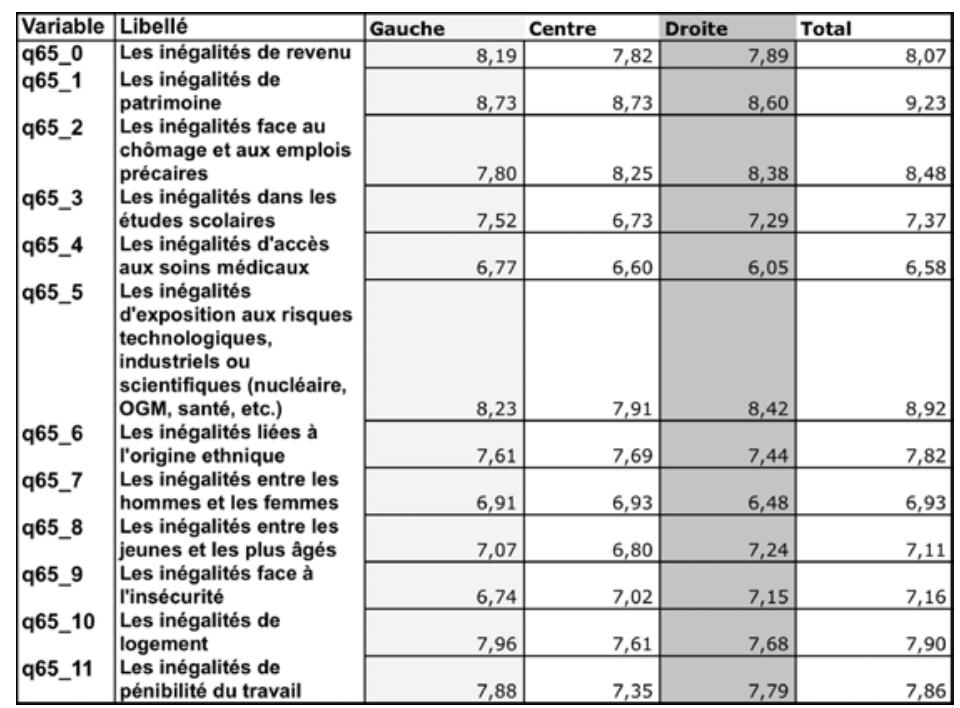

9 Le questionnaire PISJ demandait aux individus d'indiquer leurs préférences politiques, en se positionnant sur une échelle de 10 allant de gauche à droite, le 1 représentant la position la plus à gauche. Nous avons constitué trois groupes d'opinions: gauche pour les positions de 1 à 4 , centre pour les positions 5 , droite pour les positions 6 à 10. 


\section{Tableau 3}

Acceptabilité des inégalités en France selon les préférences politiques

\begin{tabular}{|c|c|c|c|c|c|}
\hline Variable & Libellé & auche & Centre & Droite & Total \\
\hline \multirow{3}{*}{$\begin{array}{l}\text { q66_0 } \\
\text { q66_1 } \\
\text { q66_2 }\end{array}$} & \multirow{6}{*}{$\begin{array}{l}\text { Les inégalités de revenu } \\
\text { Les inégalités de patrimoine } \\
\text { Les inégalités face au chômage et } \\
\text { aux emplois précaires } \\
\text { Les inégalités dans les études } \\
\text { scolaires } \\
\text { Les inégalités d'accès aux soins } \\
\text { médicaux } \\
\text { Les inégalités d'exposition aux } \\
\text { risques technologiques, industriels } \\
\text { ou scientifiques }\end{array}$} & 4,14 & 3,99 & 4,62 & 4,25 \\
\hline & & 5,36 & 25 & 5,98 & 6,07 \\
\hline & & 3,45 & 3,70 & 4,30 & 3,92 \\
\hline \multirow{3}{*}{$\begin{array}{l}\text { q66_3 } \\
\text { q66_4 } \\
\text { q66_5 }\end{array}$} & & 4,02 & 3,61 & 4,22 & 4,17 \\
\hline & & 2,95 & 3,08 & 3,76 & 3,24 \\
\hline & & 4,31 & 4,55 & 4,99 & 5,24 \\
\hline \multirow{2}{*}{$\begin{array}{l}\text { q66_6 } \\
\text { q66_7 }\end{array}$} & \multirow{6}{*}{$\begin{array}{l}\text { Les inégalités entre les hommes et } \\
\text { les femmes } \\
\text { Les inégalités entre les jeunes et } \\
\text { les plus âgés } \\
\text { Les inégalités face à l'insécurité } \\
\text { Les inégalités de logement } \\
\text { Les inégalités de pénibilité du } \\
\text { travail }\end{array}$} & 3,29 & 3,74 & 4,51 & 4,20 \\
\hline & & 3,12 & 3,45 & 3,33 & 3,32 \\
\hline \multirow{4}{*}{$\begin{array}{l}q 66 \_9 \\
q 66 \_10 \\
q 66 \_11\end{array}$} & & 3,80 & 4,09 & 3,79 & 4,07 \\
\hline & & 3,66 & 3,62 & 4,10 & 3,89 \\
\hline & & 3,37 & 3,22 & 3,96 & 3,56 \\
\hline & & 3,80 & 3,40 & 4,68 & \\
\hline
\end{tabular}

Tableau 4

V de Cramer ${ }^{10}$

\begin{tabular}{|c|c|c|c|c|c|c|}
\hline & \multicolumn{2}{|l|}{ PCS } & \multicolumn{2}{|l|}{ revenus } & \multicolumn{2}{|c|}{ opinions politiques } \\
\hline & $\checkmark$ de Cramer & chi2 & v de Cramer & & v de Cramer & \\
\hline Les inégalités de revenu & 0,102 & w. & 0,105 & w. & 0,144 & w* \\
\hline Les inégalités de patrimoine & 0,083 & & 0,08 & & 0,143 & $\cdots$ \\
\hline $\begin{array}{l}\text { Les inégalités face au chômage et aux emplois } \\
\text { précaires }\end{array}$ & 0,091 & & 0,087 & & 0,129 & .** \\
\hline Les inégalités dans les études scolaires & 0,085 & & 0,074 & & 0,14 & $\cdots$ \\
\hline Les inégalités d'accès aux soins médicaux & 0,079 & & 0,088 & & 0,139 & $\cdots$ \\
\hline $\begin{array}{l}\text { Les inégalités d'exposition aux risques } \\
\text { technologiques, industriels ou scientifiques } \\
\text { (nucléaire, OGM, santé, etc.) }\end{array}$ & 0,073 & & 0.087 & & 0,085 & \\
\hline Les inégalités liées à l'origine ethnique & 0,089 & & 0,082 & & 0,146 & $\cdots$ \\
\hline Les inégalités entre les hommes et les femmes & 0,082 & & 0,074 & & 0,098 & - \\
\hline Les inégalités entre les jeunes et les plus âgés & 0,085 & & 0,083 & & 0,121 & $\cdots$ \\
\hline Les inégalités face à l'insécurité & 0,09 & * & 0,088 & & 0,68 & \\
\hline Les inégalités de logement & $0,088^{*}$ & & 0,082 & & 0,142 & $\cdots$ \\
\hline Les inégalités de pénibilité du travail & 0,089 & - & 0.082 & & 0,145 & ... \\
\hline Les inégalités de revenu & 0,097 & "* & $\overline{0,1}$ & & 0,125 & w. \\
\hline Les inégalités de patrimoine & 0,097 & $\cdots$ & 0,106 & $\cdots$ & 0,109 & $*$ \\
\hline $\begin{array}{l}\text { Les inégalités face au chômage et aux emplois } \\
\text { précaires }\end{array}$ & 0,081 & & 0,086 & & 0,125 & *.* \\
\hline Les inégalités dans les études scolaires & 0,079 & & 0,067 & & 0,122 & $\cdots *$ \\
\hline Les inégalités d'accès aux soins médicaux & 0,079 & & 0,072 & & 0,098 & $\cdot$ \\
\hline $\begin{array}{l}\text { Les inégalités d'exposition aux risques } \\
\text { technologiques, industriels ou scientifiques } \\
\text { (nucléaire, OGM, santé, etc.) }\end{array}$ & 0.069 & & 0,061 & & 0,112 & .. \\
\hline Les inégalités liées à l'origine ethnique & 0,077 & & 0,069 & & 0,165 & $\cdots$ \\
\hline Les inégalités entre les hommes et les femmes & 0,081 & & 0,073 & & 0,135 & $\cdots$ \\
\hline Les inégalités entre les jeunes et les plus âgés & 0,083 & * & 0,079 & & 0,093 & \\
\hline Les inégalités face à l'insécurité & 0,072 & & 0,068 & & 0,081 & \\
\hline Les inégalités de logement & 0,088 & - & 0.068 & * & 0,122 & $\cdots$ \\
\hline Les inégalités de pénibilité du travail & 0,07 & & 0,077 & & 0,094 & \\
\hline
\end{tabular}

$10 * * *$ significatif au seuil de $1 \%$, ** significatif au seuil de $5 \%$. 